\title{
Diabetic Retinopathy in the Aging Population: A Perspective of Pathogenesis and Treatment
}

\author{
Sameer P Leley $\mathbb{D}^{\prime}$ \\ Thomas A Ciulla (iD ${ }^{2-4}$ \\ Ashay D Bhatwadekar (iD ${ }^{2}$ \\ 'Indiana University School of Medicine, \\ Indianapolis, IN, USA; ${ }^{2}$ Department of \\ Ophthalmology, Eugene and Marilyn Glick \\ Eye Institute, Indiana University, \\ Indianapolis, IN, USA; ${ }^{3}$ Clearside \\ Biomedical, Inc., Alpharetta, GA, USA; \\ ${ }^{4}$ Midwest Eye Institute, Indianapolis, \\ IN, USA
}

\begin{abstract}
The elderly population in the United States is projected to almost double by the year 2050. In addition, the numbers of diabetics are rising, along with its most common complication, diabetic retinopathy (DR). To effectively treat DR within the elderly population, it is essential first to consider the retinal changes that occur due to aging, such as decreased blood flow, retinal thinning, and microglial changes, and understand that these changes can render the retina more vulnerable to oxidative and ischemic damage. Given these considerations, as well as the pathogenesis of DR, specific pathways could play a heightened role in DR progression in elderly patients, such as the polyol pathway and the vascular endothelial growth factor (VEGF) axis. Current ocular treatments include intravitreal corticosteroids, intravitreal anti-VEGF agents, laser photocoagulation and surgical interventions, in addition to better control of underlying diabetes with an expanding range of systemic treatments. While using therapeutics, it is also essential to consider how pharmacokinetics and pharmacodynamics change with aging; oral drug absorption can decrease, and ocular drug metabolism might affect the dosing and delivery methods. Also, elderly patients may more likely be nonadherent to their medication regimen or appointments than younger patients, and undertreatment with anti-VEGF drugs often leads to suboptimal outcomes. With a rising number of elderly DR patients, understanding how aging affects disease progression, pharmacological metabolism, and adherence are crucial to ensuring that this population receives adequate care.
\end{abstract}

Keywords: diabetic retinopathy, aging, vascular endothelial growth factor, adherence, pharmacodynamic, pharmacokinetic

\section{Introduction}

Looking to the future, the population is aging, and there will be an increase in the number of individuals with diabetes. In 2014 it was estimated that about 415 million adults worldwide had diabetes; by 2050, this number is predicted to increase to 642 million. ${ }^{1}$ On top of that, in 2015, 1 in 9 Americans were 65 or older. By 2050 this population is predicted to increase to account for 1 in 5 Americans. ${ }^{2}$ The significance of this is that the longer people live with diabetes, the more likely they are to develop complications such as diabetic retinopathy (DR), a microvascular disease where hyperglycemia facilitates damage to the retina and can result in vision loss and blindness, DR is the leading cause of this in workingage adults living in developed nations. ${ }^{3,4}$ It is estimated that approximately $75 \%$ of patients with type 1 diabetes and $50 \%$ of patients with type 2 diabetes will develop DR. ${ }^{5}$ With the aging and diabetic populations getting ever more extensive, it is critical to determine if and how the progression of DR changes in the aging and the best strategies to treat the disease in an aging population.
Correspondence: Ashay D Bhatwadekar Department of Ophthalmology, Eugene and Marilyn Glick Eye Institute, 1160

W. Michigan Street, GK-305P,

Indianapolis, IN, 46202, USA

Tel +| 3|7-278-5075

Fax + I 317-274-2277

Email abhatwad@iupui.edu 


\section{Diabetic Retinopathy and the Aging Population}

DR is a growing problem, especially in aging diabetics. From 1990 to 2015, all causes of blindness other than DR showed decreased crude prevalence while DR increased by $7.7 \%$ in the general population. ${ }^{6}$ The prevalence of DR is also rising in the elderly. In 2007 the number of patients over 65 with DR in the United States was about 2.5 million. It is expected to almost quadruple to 9.9 million by the year $2050 .^{7}$ In addition to this DR is increasingly causing vision loss in the elderly. In adults aged 50 and older, vision impairment due to DR increased by $9.6 \% .{ }^{6}$ Looking at the predisposing factors for DR, it becomes evident why its occurrence in the elderly is rising.

Prevalence has a positive correlation with the duration of diabetes, especially with poor glycemic control. ${ }^{8}$ A study of 12,112 diabetic patients, with a mean age of 59.5 years, in Hong Kong, found that two to five years after diagnosis of diabetes, the prevalence of DR was about $26 \%$, rising to $45.2 \%$ in patients who had been diagnosed with diabetes for more than ten years. ${ }^{3}$ Severity of DR also has a positive correlation with duration of diabetes. ${ }^{8,9}$ Considering this helps to explain the increase in DR prevalence and vision impairment among the aging population.

In addition to this, DR often coincides with other microvascular diabetic complications such as diabetic nephropathy (DN). ${ }^{10}$ Both DR and DN involve endothelial dysfunction, resulting in proteinuria and DN has been found to increase the risk of cardiovascular events. ${ }^{11-13}$ Consequently, the aging diabetic becomes increasingly frail with the accumulation of comorbidities, especially in older men, and this is associated with higher mortality rates and more hospitalizations and rehospitalizations. ${ }^{14,15}$ Properly addressing DR in the elderly could reduce the burden of one comorbidity, and as such, make for less frail patients.

\section{Retinal Changes with Aging}

As with other organs and tissues, aging changes the structure of the retina. A study comparing young rat retinas to those of elderly and senile rats showed that with aging, the overall thickness of the retina decreases, and in senile retinas, there was a marked decrease in the thickness of the inner nuclear layer, with it only having 2-3 rows of cells. ${ }^{16,17}$
Ocular blood flow and its regulation are altered with senescence, and elderly retinas also show, on average, a $20 \%$ decrease in macular blood flow. ${ }^{18-20}$ These agerelated changes in perfusion may further exacerbate the well-described ocular perfusion abnormalities in diabetes, including impaired blood flow regulation. ${ }^{21,22}$ In addition to these occurrences, there are changes to the microglia of the retina. Young retinal microglia have motile cellular processes and are very responsive to injuries, whereas aged retinal microglia do not have very motile processes and respond much slower to injuries. ${ }^{23}$ Aged retinal microglia are also denser and have a smaller dendritic arbor. ${ }^{23}$ In addition to this, in young eyes, the outer retina usually is free of microglia but, with aging, they begin to proliferate into this space and come into contact with the retinal pigment epithelium (RPE); these cells can then contribute to proinflammatory responses in this space. ${ }^{23}$ At the same time, RPE production of melanin decreases while the accumulation of lipofuscin increases. ${ }^{24}$ Müller cells also undergo changes that make them more susceptible to oxidative stresses as they age; mitochondrial dysfunction is thought to play a role in this susceptibility. ${ }^{20,24}$ Alongside these microglial changes, the number of neurons within the retina also decreases with age and contributes to decreased visual acuity associated with aging. ${ }^{24}$ These structural changes, summarized in Table 1.

The retina also experiences metabolic changes with aging. Advanced glycation end products (AGEs) accumulate in the RPE and within Bruch's membrane. ${ }^{25}$ There is an accumulation of methylated metabolites like 1-methylnicotinamide (1-methylNAA), 3-methylglutarate, and 5'methylthioadenosine in the aged retina. ${ }^{24}$ The production of these metabolites requires the donation of a methyl group from S-adenosylmethionine (SAM), depleting

Table I Structural Retinal Changes with Aging

\begin{tabular}{|l|c|}
\hline $\begin{array}{l}\text { Retinal } \\
\text { Component }\end{array}$ & Change from Aging \\
\hline Thickness & Decreases, especially the Inner Nuclear Layer. ${ }^{16,17}$ \\
\hline $\begin{array}{l}\text { Macular Blood } \\
\text { Flow }\end{array}$ & Reduced by 20\% on average. \\
\hline Microglia & $\begin{array}{r}\text { Less motile, slower response time to injury, } \\
\text { denser, with a smaller dendritic arbor. }\end{array}$ \\
\hline RPE & $\begin{array}{r}\text { Decreased melanin production, increased } \\
\text { lipofuscin accumulation. }{ }^{23,24}\end{array}$ \\
\hline Müller Cells & More susceptible to oxidative stress. ${ }^{20,24}$ \\
\hline
\end{tabular}


cellular methyl group reserves which are also necessary for epigenetic modification, potentially hindering that process and contributing to structural changes in the retina. ${ }^{24}$ The increased production of 1-methylNAA from nicotinamide is also thought to inhibit the production of nicotinamide adenine dinucleotide $(\mathrm{NAD}+)$, potentially explaining the decrease in $\mathrm{NAD}+$ levels in aging retinas. ${ }^{24,26}$ All these changes help account for the natural decline in visual acuity with aging. Some of them have the potential to alter the pathogenesis of DR in the aging population.

\section{Pathogenesis of DR and the Effects of Aging}

Diabetes and the resulting hyperglycemia are the driving forces behind the progression of DR. Normally glycolysis is very tightly regulated to maintain a steady state concentration of its intermediates; in times of great metabolic demand, like ischemia, there is an increased glycolytic flux which results in a greater number of glycolytic intermediates than can be handled by the normal pathways and the excess are sent into the downstream pathways. ${ }^{27}$ Diabetic end organ complications, such as DR, develop as hyperglycemia progressively contributes to sorbitol accumulation via aldose reductase, formation of AGEs, protein kinase $\mathrm{C}$ activation, oxidative stress and angiogenic pathways. ${ }^{28} \mathrm{~A}$ number of these pathways have been thought of as links between hyperglycemia and DR, and are reviewed below. ${ }^{27,29}$

\section{Polyol Pathway}

Typically cellular glucose is handled by hexokinase in retinal cells; however, when intracellular glucose levels rise high enough to saturate hexokinase, the excess glucose gets shunted into the polyol pathway. ${ }^{27}$ Here, it is converted first into sorbitol by aldolase reductase (AR). This reaction uses up nicotinamide adenine dinucleotide phosphate (NADPH), which in turn reduces the functionality of glutathione reductase, hindering intrinsic antioxidant activity within cells. In addition to this, sorbitol cannot be transported out of the cell; this is thought to cause osmotic damage and eventually cell death potentially. ${ }^{29,30}$ The trapped sorbitol is slowly converted to fructose via sorbitol dehydrogenase (SDH); the fructose can then be phosphorylated to fructose-3-phosphate and further converted into 3-deoxyglucosone; both of which are potent glycating agents. ${ }^{29}$ Increased SDH activity also promotes the production of reactive oxidative species
(ROS). ${ }^{29}$ This is problematic and cytotoxic due to the impeded glutathione reductase activity. Within the retina, AR is localized to certain cells: pericytes, retinal endothelial cells, ganglion cells, Müller cells, RPE cells, and neurons; increased AR activity within these cells has been associated with their death. ${ }^{29,30}$ Damage to these cells could damage the retinal microvasculature and decrease visual acuity. AR activity was also measured at significantly higher levels in the vasculature of aged rats than in young rats; another observation was that the aged endothelial cells also showed significantly lower antioxidant capabilities. ${ }^{31}$ As mentioned earlier, Müller cells are at a higher risk of oxidative damage as they age, so there is potential that this pathway could play a heightened role in the progression of DR in elderly patients.

\section{AGEs}

AGEs are naturally occurring results of nonenzymatic covalent linkage, Maillard reaction, between a reducing sugar and a protein or lipid. They are observed in embryonic development and continue to form at a slow constant rate in healthy individuals; however, in people with diabetes, the rate of AGE formation is markedly increased due to the greater availability of glucose. ${ }^{29}$ AGEs are structurally and functionally different from their lipid or protein precursor. They can accumulate to cause damage when they crosslink with extracellular matrix (ECM) components, which can decrease elasticity. ${ }^{32}$ AGEs can also bind to cell surface receptors for AGEs (RAGE); within the retina, these receptors are found in endothelial cells, pericytes, microglia, Müller cells, and RPE, and their expression increases in the diabetic state. ${ }^{32}$ This AGE-RAGE binding causes signaling cascades that result in pro-oxidant, proinflammatory events, like increased vascular endothelial growth factor (VEGF) secretion, which can further damage the retinal microvasculature and the blood-retinal barrier (BRB). ${ }^{25,29,32,33}$ It is believed that AGE accumulation within retinal pericytes increases significantly in the diabetic state and results in the apoptosis of these cells due to a resultant increase in production of ROS; pericyte loss is considered to be one of the first signs of DR. ${ }^{32}$

\section{Protein Kinase C (PKC) Activation}

$\mathrm{PKC}$ is a serine/threonine kinase that is activated by diacylglycerol (DAG); hyperglycemia causes increased de novo production of DAG. ${ }^{29}$ The PKC family functions as members of signaling cascades associated with $G_{\alpha o}$ and $\mathrm{G}_{\alpha \mathrm{q}}$ G-protein coupled receptors. Out of this family of 
kinases, the beta isoform (PKC $\beta$ ) is the principal active isozyme, especially in vascular tissue, during hyperglycemia. The $\beta 1 / 2$ isoform is closely associated with $D R$, and its expression is upregulated in the diabetic state. ${ }^{29,34}$ Increased PKC activation within the retina has been observed to have multiple effects, including increased endothelial permeability, altered hemodynamics, increased VEGF secretion, and increased leukostasis. ${ }^{29,34}$ The increased activation also alters the composition of the retinal ECM resulting in changes in the synthesis of component proteins and endothelial and leukocyte dysfunction, which can lead to occluded capillaries. ${ }^{29}$

\section{Hexosamine Pathway}

Fructose-6-Phosphate (F-6-P) is an intermediate of the glycolytic pathway, which in the healthy state is predominantly converted into fructose-1,6-bisphosphate (F-1,6-BP) and further broken down to generate pyruvate. Under hyperglycemic conditions, F-6-P buildup can overwhelm the body's capacity to generate F-1,6-BP and the excess is shunted into the hexosamine pathway and converted to N-acetyl glucosamine-6-phosphate (GlucN-6-P) by glutamine fructose-6-phosphate-amido transferase (GFAT). ${ }^{27,35}$ GlucN-6-P is then converted into uridine5-diphosphate (UDP)-N-acetyl glucosamine (UDPGlcNAc), a precursor for many biologically required amino sugars. ${ }^{27}$ Under normoglycemic conditions, this pathway only handles a small percentage of total glucose metabolism, but it is highly upregulated to handle the excess F-6-P present in hyperglycemia; this can result in excess protein glycosylation, which can, in turn, alter gene expression and cellular functions. ${ }^{27}$ Hyperactivity of this pathway has been shown to increase cell death within retinal neurons and ganglion cells. ${ }^{27}$ It can also glycosylate p53 in retinal pericytes; this has been linked with increased pericyte apoptosis and vascular dysfunction. ${ }^{27}$

\section{VEGF}

Inflammatory responses are among the body's primary responses to diabetes, and this is also seen in the retina with $\mathrm{DR} .{ }^{5}$ One of the ways this response is initiated in the retina is through the release of VEGF. The VEGF family of proteins includes VEGF-A, VEGF-B, VEGF-C, VEGF-D, VEGF-E, and placental growth factor (PGF). VEGF-A binds to VEGF receptor 1 (VEGFR1 or Flt-1) and VEGF receptor 2 (VEGFR2 or KDR/Flk-1), which mediates angiogenesis and vascular leakage. VEGF-A, initially termed vascular permeability factor (and often referred to simply as
"VEGF"), promotes angiogenesis and vascular permeability, contributing to the breakdown of the inner BRB, retinal edema and microhemorrhages. ${ }^{5,20,29,36,37}$ it is believed that this might be an attempt by the body to keep cells viable in the presence of the increase in metabolic activity associated with diabetes. ${ }^{5}$ While promoting vascularity at the site of inflammation, VEGF exacerbates the inflammatory reaction by allowing higher levels of proinflammatory cells modulators. The inflammatory response might be beneficial in the short term; it can be damaging if maintained for an extended period. Prolonged metabolic stresses and inflammation can cause permanent damage to the retina, including edema, invasion of immune cells, and scarring. ${ }^{5}$

As described in Figure 1, each of the pathways discussed above can play a role in DR, and it is likely a synergy between them that allows the disease to progress and worsen. Looking at the elderly population, some pathways of note are the polyol pathway and the AGE pathway due to the ways in which they can be sources of oxidative stress within retinal cells; taking advantage of the fact that the aged retina's defenses against oxidative stress have been weakened due to the structural and metabolic changes associated with aging. Also of note is the VEGF pathway which could exacerbate the already reduced blood flow in the elderly retina.

\section{Clinical Studies of DR in Young and Aging Populations}

To date, there have been only a few clinical studies focused on DR in the aging population. One such study focused on screening habits in Iran found that prevalence of DR increased with age from the ages of 55 to 74; in the 55-59 age group, the prevalence was around $1.0 \%$, and it increased with each subsequent group peaking at $8.2 \%$ in the 70-74 age group, the 75 and older group did not follow this trend with a prevalence of $3.4 \%{ }^{38}$ Another study in Australia found that in elderly patients, mean age 64.9, severe nonproliferative DR (NPDR) and proliferative DR (PDR) were associated with higher levels of depression. ${ }^{39}$ This could present another complication in the care of elderly patients with DR. In addition to these findings, further analysis of the Action to Control Cardiovascular Risk in Diabetes (ACCORD) Trial found that DR was associated with increased risk for cognitive decline in type 2 diabetics. ${ }^{40}$ In some instances, there have been studies looking at other facets of diabetes in elderly populations that have had findings that pertain to DR. One study, SUSTAIN 6, aimed at ascertaining the potential 


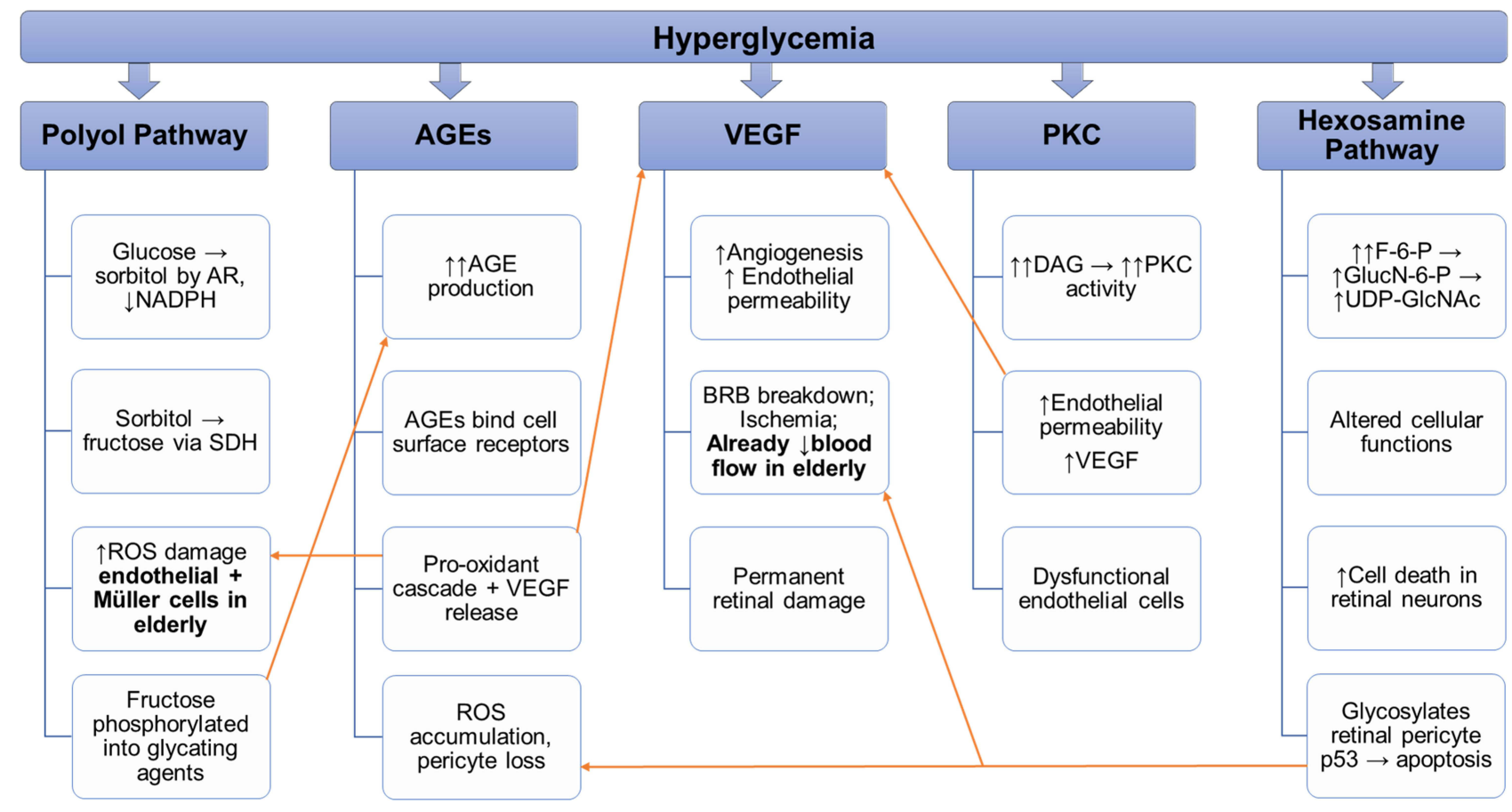

Figure I Interconnected pathways of DR and areas of susceptibility for the elderly.

Notes: All the above pathways contribute to DR progression in unique ways. They have some mechanisms that can exacerbate the effects of other pathways. Namely, many of the pathways increase VEGF production, which could impact the role of the VEGF pathway and take advantage of some age-related changes that leave the retina more susceptible to DR damage.

benefits of glucagon-like peptide 1 (GLP-1) found that while these drugs might indeed have protective effects against the cardiovascular complications of diabetes, in patients over 50 , there was a significantly higher risk of developing new or worsening DR in those treated with the GLP-1 agonist semaglutide than those receiving the placebo. ${ }^{41}$ The reason for this occurrence was explained via post-hoc analysis, which revealed that it was due to a known cause: early DR can worsen with sudden and significant reductions in hemoglobin $\mathrm{A} 1 \mathrm{c}$ (HbA1c) in patients who had poor glycemic control in the first place. ${ }^{42}$ For patients whose DR is moderate NPDR or less severe, strict glycemic control, maintaining HbAlc concentrations below 7\%, can slow disease progression and reduce severe disease risk. ${ }^{43}$ The increasing use of telemedicine has allowed for better study of DR in aging populations; one such study, mean age 66 , found links between high-density lipoprotein cholesterol (HDL-C) levels and the risk for DR and PDR. ${ }^{44}$ The mechanisms behind this link between HDL-C and DR need further study; although high-density lipoproteins (HDL) have been shown to be beneficial, when these molecules contain cholesterol, HDL-C, they have little clinical benefit and may even be harmful. ${ }^{45}$ The use of telemedicine opens the door for even more research on DR in the elderly population. The telemedicine-based studies have also allowed for better study of the comorbidities often associated with DR in the elderly like DN. ${ }^{44,46}$

Studies of DR in younger populations have also shed some light on the changes that occur with DR in older populations. For example, they have shown that younger patients are more likely to develop PDR than older patients. ${ }^{8,47}$ It has also been found that even when older patients develop PDR, they are less likely to suffer from postoperative complications than young patients if they require a vitrectomy; this is thought to be attributed to the fact that younger patients have greater vitreoretinal adhesion and more vasculature in that region. ${ }^{47}$ One finding that does not change between the two populations is that in both groups, the risk of developing DR increases with the duration of diabetes. ${ }^{48}$ The one exception to this is that young patients with type 1 diabetes are more likely to develop DR than those with type 2 diabetes and they tend to develop it at a faster rate. ${ }^{49}$

\section{Current and Emerging Treatments}

Currently, the best intervention for DR is detection at an early stage which may facilitate prompt treatment and 
prevent vision loss. ${ }^{50}$ Early and frequent screening is vital as many patients are asymptomatic in the early stages of the disease. ${ }^{51}$ The current standard for screening is an ophthalmic exam with pupillary dilation and retinal evaluation. ${ }^{51}$ Yet this is still a challenge, especially in lowmiddle income countries where there may be as few as one ophthalmologist per million people, approximately $1 \%$ of the proportion in the United States, and most of these ophthalmologists often live in urban areas, leaving rural patients further underserved. ${ }^{52}$ However, advances in technology facilitate teleophthalmology, a form of telemedicine, with remote color fundus cameras capable of sending high-quality images to retinal specialists for evaluation. ${ }^{51}$ The importance and usefulness of telemedicine, especially in the elderly, has been highlighted by the COVID-19 pandemic; studies have shown that teleophthalmology is adequate for glaucoma diagnosis and recent advances in smartphone imaging devices have led to significant increases in the number of patients screened for DR using telemedicine ${ }^{53}$ Advances in artificial intelligence show promise in accelerating this process, potentially providing instantaneous feedback on whether the patient requires follow up with an ophthalmologist and even determining which treatment may be indicated. ${ }^{51,54}$ These technological advances and the widespread use of telemedicine could also improve access to DR screening for low-middle income countries.

Therapeutically, corticosteroids were utilized early in the treatment of DR. Currently, two intravitreal corticosteroid therapies are approved by the US Food and Drug Administration (FDA) for the treatment of DME: OZURDEX $^{\circledR}(0.7 \mathrm{mg}$ dexamethasone intravitreal implant, Allergan) and ILUVIEN ${ }^{\circledR}$ (0.19 mg fluocinolone acetonide intravitreal implant, Alimera Sciences). Corticosteroid mechanism of action in the treatment of macular edema is multifactorial and complex, involving interaction with corticosteroid hormone receptor which can alter gene expression and synthesis of inflammatory and antiinflammatory proteins, improvement of blood-retinal barrier permeability by increased expression of retinal endothelial junction proteins and stabilization of lipid membranes, as well as inhibition of macrophage migration, and down regulation of eicosanoid production. Corticosteroids also decrease elaboration of VEGF by proinflammatory mediators. However, when administered as currently approved via the intravitreal route, there is an increased risk of ocular hypertension and glaucoma. ${ }^{55}$
The current standard of care for the treatment of DME is intravitreal anti-VEGF therapy. Currently, two intravitreal anti-VEGF agents are approved by the US FDA for the treatment of DME: EYLEA ${ }^{\circledR}$ (aflibercept, Regeneron) and LUCENTIS ${ }^{\circledR}$ (ranibizumab, Genentech). Also, one agent is routinely used intravitreally off-label, AVASTIN $^{\circledR}$ (bevacizumab, Genentech). Initially intended for diabetic macular edema, anti-VEGF agents have also shown promise in treating DR. ${ }^{36,56}$ These agents bind VEGF-A, which normally activates the VEGF receptor (VEGFR)-2, to mediate angiogenesis and vascular leakage. The use of these agents has been widely adopted in the industrialized world, given their efficacy. However, these agents have a short half-life and require monthly or bi-monthly intraocular injections. In addition, these injections can increase the risk of developing endophthalmitis due to the introduction of microorganisms, and this risk is exacerbated by the need for frequent intravitreal injection. ${ }^{36}$

Laser photocoagulation, practiced for decades before the widespread adoption of anti-VEGF therapy, remains another treatment option for DR. Laser photocoagulation was found to reduce the risk of moderate vision loss by up to $50 \%$ in the Early Treatment Diabetic Retinopathy Study; the mechanism of action is complex and multifactorial but involves stimulation of RPE cells and reduction of metabolic requirements. However, potential side effects of laser photocoagulation include loss of RPE and photoreceptors leading to paracentral scotomas after macular photocoagulation for macular edema or reduced visual field and nyctalopia after pan-retinal photocoagulation for PDR $^{36}$ For PDR complicated by vitreous hemorrhage, pars plana vitrectomy with laser photocoagulation remains an option; traction retinal detachment can be addressed by peeling causative membranes that result from neovascularization. Pars plana vitrectomy with the stripping of macular membranes is sometimes used in cases of refractory diabetic macular edema.

There are several promising therapeutics under development to help stop or slow the progression of DR. AR inhibitors (ARIs) are one example that work by inhibiting the polyol pathway; as discussed earlier, the overactivity of this pathway increases oxidative stress within cells. Early animal studies in spontaneously diabetic torii (SDT) rats have shown that the ARI ranirestat may inhibit upregulation in AR activity and have neuroprotective effects when used in the early stages of DR. ${ }^{17}$ Glucose transporter 1 (GLUT-1) siRNA has also shown promise in 
reducing retinal glucose concentrations, by reducing the number of GLUT-1 channels, and protecting photoreceptor cells from hyperglycemic damage in diabetic state mice. ${ }^{57}$ This reduced retinal glucose concentration could also reduce the use of the alternate glucose metabolic pathways associated with the pathogenesis of DR. Another promising avenue is the modification of sirtuin 1 (Sirt1) expression. Sirt1 is a deacetylase known to be downregulated in DR. Animal studies in mice have found that the Sirt1 overexpression slowed the progression of DR, and Sirt1 diabetic mice had fewer acellular capillaries than the wild type diabetic. ${ }^{58}$ This Sirtl downregulation could result in altered gene expression that favors the progression of DR, and inducing its overexpression could mitigate those effects. PKC inhibition has also shown promise in animal studies, helping prevent the breakdown of the BRB and limiting retinal neuropathy; however, it has only had significant results in human studies when used in combination with photocoagulation. ${ }^{59}$ There are also studies looking at alternatives to anti-VEGF therapeutics. Although there have not yet been any clinical trials, gene therapy to insert antiVEGF genes has the potential to prevent the leaky angiogenesis associated with DR. ${ }^{59,60}$

In addition to these preclinical candidates, there are a few potential therapies in clinical trials, or have already been approved to treat other aspects of diabetes. One such therapy is sodium-glucose cotransporter 2 (SGLT2) inhibition which has had demonstrated nephroprotective and cardioprotective benefits. ${ }^{61,62}$ SGLT2 inhibitors also hold promise as a potential treatment for DR because of their ability to protect retinal pericytes from hypoglycemic damage and correct systemic hyperglycemia by reducing the reabsorption of glucose in the proximal convoluted tubule of the nephron. ${ }^{63,64}$

Another promising treatment involves the interaction between angiopoietins and tyrosine receptor kinases. Angiopoietin-1 (Ang1) binds to the tyrosine receptor kinase (Tie2), activating vascular maturation; Angiopoietin-2 (Ang2) deactivates Tie2 causing vascular destabilization. ${ }^{59}$ Increased Ang2 activity could synergistically affect VEGF and result in a more significant number of leaky vessels in the diabetic retina. Several new drugs in development aim to inhibit Ang2 or Ang2 and VEGF to restore Tie2 activity. ${ }^{59}$

Recently, one such agent, Faricimab, a bispecific antibody for both VEGF-A and Ang2, was assessed in two global phase 3 clinical trials. These studies demonstrated that Faricimab administered intravitreally every 8 to 16 weeks was non-inferior to standard-of-care aflibercept administered intravitreally every 8 weeks. ${ }^{65}$ New treatments with prolonged durability or enhanced efficacy, combined with early screening, could dramatically change the progression of DR and considerably decrease the development of severe forms of the disease. While these new and existing treatments do hold promise, some considerations must be taken when looking at elderly patients.

\section{Challenges for the Aging Population}

Prescribing medication within an elderly population can present several unique problems. It is thought that pharmacodynamic and pharmacokinetics might change with aging and alter how drugs are absorbed and metabolized. Another concern with this population is an increased medication nonadherence rate, where patients do not take their medications per their treatment plan.

\section{Pharmacokinetic and Pharmacodynamic Changes}

There is a decrease in a mucosal surface area within the gastrointestinal system with aging, and it is thought that this might result in a decreased rate of gastrointestinal drug absorption. ${ }^{66}$ It has also been noted that drug absorption is diminished to a greater degree in malnourished, underweight, elderly patients than those who are wellnourished. ${ }^{67}$ Another age-related change is that gastric acid secretion can decrease with aging, and the use of proton pump inhibitors can exacerbate this decrease; the decrease could reduce the absorption of drugs that require an acidic environment to pass through cellular membranes. ${ }^{68}$ They might not experience any therapeutic benefits from a dose that would otherwise be effective in a patient with normal absorption. This decreased absorption could require a different dosing schedule or a different delivery method for these patients to receive benefits from their medications.

For DR, current standard-of-care intravitreal injections are also susceptible to pharmacokinetic variation with aging; for example, it has been found that aging causes an increase in proteases within the vitreous. ${ }^{69}$ A potential implication of this would be that certain protein drugs like antibodies could undergo a pseudo-first-pass effect metabolism within the vitreous. However, more research is needed to determine if this is indeed the case or if the increased protease levels have no significant impact on 
protein drugs delivered via intravitreal injections. ${ }^{69}$ There are also aging-related changes that could affect the volume of distribution of certain medications within the eye. Some medicines, such as tyrosine kinase inhibitors, can bind to melanin, and this can prolong the effect of the drug in melanin-rich tissue such as the RPE and choroid. ${ }^{69}$ As the eye ages, the amount of melanin within the RPE decreases, and the amount of melanin within the choroid increases, and this could shift the melanin binding drugs out of the RPE and into the choroidal tissues. ${ }^{69}$

Cellular receptors have also been shown to have changing sensitivity to ligands with aging, which could affect the efficacy of a drug based on the age of the patient. ${ }^{70}$ For example, elderly patients are more sensitive to opioids and certain anesthetics than younger patients and less sensitive to the $\beta_{2}$ receptor agonist salbutamol and the $\beta_{2}$ receptor antagonist propranolol. ${ }^{71,72}$ A change in efficacy could also cause a standard dose not to have therapeutic effects, or it could result in the dose having too much effect and crossing the border into toxic effects. The elderly also tend to have increased body fat and decreased total body water, which can lead to increased volume of distribution (Vd) for lipophilic drugs and a decreased $\mathrm{Vd}$ for hydrophilic drugs. ${ }^{73}$ That could result in lipophilic drugs being sequestered within the fat stores and being released into the plasma at a later time. While these aging changes potentially alter how drugs are absorbed and distributed within the body, the way they are metabolized does not seem to undergo much of a change. Although hepatic mass and circulation decrease with aging, hepatic drug metabolism appears to be preserved in the elderly up to age $80 .^{73}$ Whereas hepatic metabolism might not be affected, renal clearance is known to decrease with age. ${ }^{74}$ That could also have toxic effects if drug metabolites have toxic effects with prolonged exposure. The above parameters require consideration when prescribing medications to elderly patients and they could affect the treatment options for elderly patients with DR.

\section{Adherence}

Non-adherence to the medication has been observed as a longstanding problem within the diabetic community in general, and it has also been noticed as a problem within the elderly population. ${ }^{75}$ One implication of poor adherence in diabetics is that it is associated with poor glycemic control. ${ }^{76}$ Poor glycemic control and nonadherence to medicine in diabetics, especially elderly diabetics, can be a dangerous combination. An Ethiopian study of 311 patients with diabetes found that patients over the age of 60 were five times more likely to develop DR than those under 60 , patients with poor adherence were three times more likely to develop DR than those who were adherent, and those with poor glycemic control were nine times more likely to develop DR than those with good glycemic control. ${ }^{77}$ A study examining the adherence within the Atherosclerosis Risk in Communities Study, average participant age 75.4 , found that 2119 participants, or about $40 \%$, reported intermediate to poor compliance with their medication; of these participants, $75 \%$ of them said their non-adherence was due to poor memory. ${ }^{78}$ Although these results look daunting, there were some positive takeaways from the study. Patients in good physical and mental health as well as those who were "very satisfied with the care," were significantly correlated with higher drug adherence. ${ }^{78}$

Other factors have also been studied when looking at drug adherence within the elderly. Functional health literacy, or the ability to read and understand the instructions on a prescription label, is significantly lower among elderly populations, making it a contributing factor to the higher rates of drug nonadherence in this population. ${ }^{79}$ Health issues such as decreased visual acuity and dexterity are also associated with lower adherence. ${ }^{79}$ This is especially a problem in elderly patients with DR, particularly those with advanced disease and severe vision loss. Dosing frequency has also been shown to play a role. Drug regimens that call for administration once or twice a day have a higher adherence rate than drugs administered thrice daily or more frequently. ${ }^{76,79}$ Cost of medication has also been shown to play a factor in drug nonadherence with the elderly. ${ }^{80}$ This is especially a problem in low-middle income countries where patients often have to pay out of pocket for diabetes care, and there are lower rates of adherence compared to high-income countries. ${ }^{81}$ The elderly population has a higher rate of dementia, and this can cause a two to a three-fold higher chance of being nonadherent to their medication. ${ }^{82}$ Each of these factors presents a potential hurdle to seeing therapeutic benefits of prescribed medications in this population. These studies warrant communication between providers and patients about the importance of taking medications and taking them on time and efforts made to simplify the process of taking the medication for the patient.

Another type of nonadherence is patients being lost to follow up, where patients fail to show up for their scheduled appointments. This has been seen in the general 
diabetic population in regard to the annual diabetic eye exam. A study of 4072 patients age 20 and older who selfreported having diabetes found that about $36.6 \%$ of them were nonadherent to the recommendation for annual diabetic eye exams; one interesting finding of this study was that younger adults, age 30 to 35 years old, had a higher rate of nonadherence at $59 \%$ compared to those over 65 who had rates of nonadherence at $30 \%$ or lower. ${ }^{83}$ It is possible that one reason for this level of nonadherence to annual diabetic eye exams is that patients with diabetes might be unaware of DR and their increased risk for developing it or the need for annual eye exams. One study found that out of 7395 diabetic participants, only about half of them knew about the need for annual exams. Most participants did not know the reasoning behind needing the eye exams. ${ }^{84}$ When it comes to patients with DR, however, there does appear to be an increase in appointment nonadherence with aging. An Austrian study found that out of 423 patients being treated for $\mathrm{DR}$, with either intravitreal injections, photocoagulation, or a combination of the two, 122 patients, or about $28.8 \%$, were lost to follow up for at least six months. ${ }^{85}$ The study also found that long term loss to follow up was significantly higher in patients over 70 and those who needed assistance in traveling to their appointments; a resultant multivariate model found that patients of advanced age were 1.68 times more likely to be lost than younger patients and patients needing assistance traveling were 2.05 times more likely to be lost to follow up. ${ }^{85}$ These patients would be at risk of their DR worsening as any disease changes would go unnoticed by their doctors and they would not be able to receive any treatments that might prevent this disease progression. A study looking at $59 \mathrm{PDR}$ patients treated with intravitreal injections or photocoagulation, who were lost to follow up for at least six months, found that both the injection and photocoagulation groups showed a significant decrease in visual acuity when comparing the visit before being lost to the return visit. ${ }^{86}$ For diabetic macular edema patients undergoing treatment with anti-VEGF agents, studies using large databases have shown that reduced treatment intensity yields suboptimal visual acuity outcomes. ${ }^{87,88}$ As with nonadherence to medication, there is a need for better patient education and clear communication to ensure that patients are aware of the need for annual eye exams and follow-up care as well as the rationale behind those exams.

\section{Conclusion}

DR in the aging population presents a growing problem, one which is compounded by other aging-associated issues. The number of diabetics and the number of older adults in the global population are both projected to grow in the next 30 years. With DR as the leading cause of vision loss in working-age people today, it is essential to understand how DR affects aging populations as today's working population will become tomorrow's aged population. This is especially important considering that the duration of diabetes has a positive correlation with the risk of developing DR. The hyperglycemia brought on by diabetes induces a variety of changes in the retina, particularly in the upregulation of multiple glucose metabolism pathways which can have cytotoxic results. The changes in the structure of the retina that come about with aging could affect these pathways and alter the progression of DR. To treat these aging diabetics properly, further work is needed to determine the extent to which the retinal changes of aging do affect the progression of DR so that new therapeutics can be developed if required, and existing therapies can be modified to account for any age-related changes. As of now, there are a few treatment options for DR, including anti-VEGF drugs and laser photocoagulation, and there are promising new therapeutics still under development. Even with these advances, elderly patients present with several challenges when it comes to treatment with medications.

Pharmacokinetic and pharmacodynamic changes with aging can affect how drugs are metabolized or how they bind to their targets. This can result in the prescribed doses not having a therapeutic effect or even possibly crossing the threshold for toxic effects and causing harm to these patients. Elderly patients also have a higher chance of being nonadherent to their medications or appointments for a variety of reasons. Treatment plans for elderly DR patients should take this into account to ensure that patients are getting therapeutic benefits from their medications. To comprehensively address the growing number of elderly patients with DR, there is also a need for more studies, especially clinical trials, that determine the exact relationship between aging-related changes in the retina and the progression of DR.

\section{Disclosure}

Thomas A Ciulla is an employee and equity holder of Clearside Biomedical, Inc and Ashay D Bhatwadekar is an 
ad hoc pharmacist at PCA Pharmacy. The authors report no other conflicts of interest in this work.

\section{References}

1. Zheng Y, Ley SH, Hu FB. Global aetiology and epidemiology of type 2 diabetes mellitus and its complications. Nat Rev Endocrinol. 2018;14(2):88-98. doi:10.1038/nrendo.2017.151

2. Halaweish I, Alam HB. Changing demographics of the american population. Surg Clin North Am. 2015;95(1):1-10. doi:10.1016/j. suc.2014.09.002

3. Lee KMC, Sum WMR. Prevalence of diabetic retinopathy in patients with recently diagnosed diabetes mellitus. Clin Exp Optom. 2011;94 (4):371-375. doi:10.1111/j.1444-0938.2010.00574.x

4. Hendrick AM, Gibson MV, Kulshreshtha A. Diabetic retinopathy. Prim Care. 2015;42(3):451-464. doi:10.1016/j.pop.2015.05.005

5. Cohen SR, Gardner TW. Diabetic retinopathy and diabetic macular edema. Dev Ophthalmol. 2015;55:137-146. doi:10.1159/000438970

6. Flaxman SR, Bourne RRA, Resnikoff S, et al. Global causes of blindness and distance vision impairment 1990-2020: a systematic review and meta-analysis. Lancet Glob Health. 2017;5(12):e1221e1234. doi:10.1016/S2214-109X(17)30393-5

7. Paulus YM, Gariano RF. Diabetic retinopathy: a growing concern in an aging population. Geriatrics. 2009;64(2):16-20.:

8. Massin P, Kaloustian E. The elderly diabetic's eyes. Diabetes Metab. 2007;33(SUPPL. 1):S4. doi:10.1016/S1262-3636(07)80052-8

9. Scanlon PH, Aldington SJ, Stratton IM. Epidemiological issues in diabetic retinopathy. Middle East Afr J Ophthalmol. 2013;20 (4):293-300. doi:10.4103/0974-9233.120007

10. Jawa A, Kcomt J, Fonseca VA. Diabetic nephropathy and retinopathy. Med Clin North Am. 2004;88(4):1001-1036. doi:10.1016/j.mcna.20 04.04.012

11. Minutolo R, Gabbai FB, Provenzano M, et al. Cardiorenal prognosis by residual proteinuria level in diabetic chronic kidney disease: pooled analysis of four cohort studies. Nephrol Dial Transplant. 2018;33(11):1942-1949. doi:10.1093/ndt/gfy032

12. Sasso FC, Chiodini P, Carbonara O, et al. High cardiovascular risk in patients with type 2 diabetic nephropathy: the predictive role of albuminuria and glomerular filtration rate. The NID-2 Prospective Cohort Study. Nephrol Dial Transplant. 2012;27(6):2269-2274. doi:10.1093/ndt/gfr644

13. Minutolo R, Sasso FC, Chiodini P, et al. Management of cardiovascular risk factors in advanced type 2 diabetic nephropathy: a comparative analysis in nephrology, diabetology and primary care settings. J Hypertens. 2006;24(8):1655-1661. doi:10.1097/01.hjh.00 00239303.93872 .31

14. Marcucci M, Franchi C, Nobili A, et al. Defining aging phenotypes and related outcomes: clues to recognize frailty in hospitalized older patients. J Gerontol a Biol Sci Med Sci. 2017;72(3):395-402. doi:10.1093/gerona/glw188

15. Corrao S, Santalucia P, Argano C, et al. Gender-differences in disease distribution and outcome in hospitalized elderly: data from the REPOSI study. Eur J Intern Med. 2014;25(7):617-623. doi:10. 1016/j.ejim.2014.06.027

16. Mohamed MEI, El-Shaarawy EAA, Youakim MF, Shuaib DMA, Ahmed MM. Aging changes in the retina of male albino rat: a Histological, Ultrastructural and Immunohistochemical Study. Folia Morphol. 2019;78(2):237-258. doi:10.5603/FM. a2018.0075

17. Toyoda F, Tanaka Y, Ota A, et al. Effect of ranirestat, a new aldose reductase inhibitor, on diabetic retinopathy in SDT rats. $J$ Diabetes Res. 2014;2014:1-7. doi:10.1155/2014/672590

18. Harris A, Harris M, Biller J, et al. Aging affects the retrobulbar circulation differently in women and men. Arch Ophthalmol. 2000;118(8):1076-1080. doi:10.1001/archopht.118.8.1076
19. Harris A, Ciulla TA, Chung HS, Martin B. Regulation of retinal and optic nerve blood flow. Arch Ophthalmol. 1998;116(11):1491-1495. doi:10.1001/archopht.116.11.1491

20. Nag TC, Wadhwa S. Ultrastructure of the human retina in aging and various pathological states. Micron. 2012;43(7):759-781. doi:10.10 16/j.micron.2012.01.011

21. Ciulla TA, Harris A, Latkany P, et al. Ocular perfusion abnormalities in diabetes. Acta Ophthalmol Scand. 2002;80(5):468-477. doi:10.1034/j.1600-0420.2002.800503.x

22. Shoshani Y, Harris A, Shoja MM, et al. Impaired ocular blood flow regulation in patients with open-angle glaucoma and diabetes. Clin Exp Ophthalmol. 2012;40(7):697-705. doi:10.1111/j.1442-9071.20 12.02778.x

23. Ma W, Wong WT. Aging changes in retinal microglia and their relevance to age-related retinal disease. Adv Exp Med Biol. 2016;854:73-78. doi:10.1007/978-3-319-17121-0_11

24. Wang Y, Grenell A, Zhong F, et al. Metabolic signature of the aging eye in mice. Neurobiol Aging. 2018;71:223-233. doi:10.1016/j. neurobiolaging.2018.07.024

25. Nagaraj RH, Linetsky M, Stitt AW. The pathogenic role of Maillard reaction in the aging eye. Amino Acids. 2012;42(4):1205-1220. doi:10.1007/s00726-010-0778-x

26. Jadeja RN, Thounaojam MC, Bartoli M, Martin PM. Implications of NAD+ metabolism in the aging retina and retinal degeneration. Oxid Med Cell Longev. 2020;2020:1-12. doi:10.1155/2020/ 2692794

27. Yumnamcha T, Guerra M, Singh LP, Ibrahim AS. Metabolic dysregulation and neurovascular dysfunction in diabetic retinopathy. Antioxidants. 2020;9(12):1-22. doi:10.3390/antiox9121244

28. Ciulla TA, Amador AG, Zinman B. Diabetic retinopathy and diabetic macular edema: pathophysiology, screening, and novel therapies. Diabetes Care. 2003;26(9):2653-2664. doi:10.2337/ diacare.26.9.2653

29. Tarr JM, Kaul K, Chopra M, Kohner EM, Chibber R. Pathophysiology of diabetic retinopathy. ISRN Ophthalmol. 2013;2013:1-13. doi:10.1155/2013/343560

30. Lorenzi M. The polyol pathway as a mechanism for diabetic retinopathy: attractive, elusive, and resilient. Exp Diabetes Res. 2007;2007:61038. doi:10.1155/2007/61038

31. Hallam KMC, Li Q, Ananthakrishnan R, et al. Aldose reductase and AGE-RAGE pathways: central roles in the pathogenesis of vascular dysfunction in aging rats. Aging Cell. 2010;9(5):776-784. doi:10.1111/j.1474-9726.2010.00606.x

32. Xu J, Chen LJ, Yu J, et al. Involvement of advanced glycation end products in the pathogenesis of diabetic retinopathy. Cell Physiol Biochem. 2018;48(2):705-717. doi:10.1159/000491897

33. Stitt AW. AGEs and diabetic retinopathy. Invest Ophthalmol Vis Sci. 2010;51(10):4867-4874. doi:10.1167/iovs.10-5881

34. Aiello LP. The potential role of PKC $\beta$ in diabetic retinopathy and macular edema. Surv Ophthalmol. 2002;47:S263-S269. doi:10.1016/ S0039-6257(02)00391-0

35. Brownlee M. The pathobiology of diabetic complications: a unifying mechanism. Diabetes. 2005;54:1615-1625. doi:10.2337/diabetes.54. 6.1615

36. Wang W, Lo ACY. Diabetic retinopathy: pathophysiology and treatments. Int J Mol Sci. 2018;19(6). doi:10.3390/ijms19061816

37. Rudraraju M, Narayanan SP, Somanath PR. Regulation of blood-retinal barrier cell-junctions in diabetic retinopathy. Pharmacol Res. 2020;161:105115. doi:10.1016/j.phrs.2020.105115

38. Hashemi H, Khabazkhoob M, Nabovati P, et al. The prevalence of age-related eye disease in an elderly population. Ophthalmic Epidemiol. 2017;24(4):222-228. doi:10.1080/09286586.2016.1270335

39. Rees G, Xie J, Fenwick EK, et al. Association between diabetes-related eye complications and symptoms of anxiety and depression. JAMA Ophthalmol. 2016;134(9):1007-1014. doi:10. 1001/jamaophthalmol.2016.2213 
40. Hugenschmidt CE, Lovato JF, Ambrosius WT, et al. The cross-sectional and longitudinal associations of diabetic retinopathy with cognitive function and brain MRI findings: the Action to Control Cardiovascular Risk in Diabetes (ACCORD) trial. Diabetes Care. 2014;37(12):3244-3252. doi:10.2337/dc14-0502

41. Marso SP, Bain SC, Consoli A, et al. Semaglutide and cardiovascular outcomes in patients with type 2 diabetes. $N$ Engl J Med. 2016;375 (19):1834-1844. doi:10.1056/nejmoa1607141

42. Singh RP, Elman MJ, Singh SK, Fung AE, Stoilov I. Advances in the treatment of diabetic retinopathy. J Diabetes Complications. 2019;33 (12):107417. doi:10.1016/j.jdiacomp.2019.107417

43. Liu Y, Li J, Ma J, Tong N. The threshold of the severity of diabetic retinopathy below which intensive glycemic control is beneficial in diabetic patients: estimation using data from large randomized clinical trials. J Diabetes Res. 2020;2020. doi:10.1155/2020/8765139.

44. Sasso FC, Pafundi PC, Gelso A, et al. High HDL cholesterol: a risk factor for diabetic retinopathy? Findings from NO BLIND Study. Diabetes Res Clin Pract. 2019;150:236-244. doi:10.1016/j.diabres. 2019.03.028

45. Primer KR, Psaltis PJ, Tan JTM, Bursill CA. The role of high-density lipoproteins in endothelial cell metabolism and diabetes-impaired angiogenesis. Int J Mol Sci. 2020;21(10):3633. doi:10.3390/ ijms21103633

46. Sasso FC, Pafundi PC, Gelso A, et al. Relationship between albuminuric $\mathrm{CKD}$ and diabetic retinopathy in a real-world setting of type 2 diabetes: findings from No Blind Study. Nutr Metab Cardiovasc Dis. 2019;29(9):923-930. doi:10.1016/j.numecd.2019.05.065

47. Chen HJ, Wang CG, Dou HL, Feng XF, Xu YM, Ma ZZ. Effect of intravitreal ranibizumab pretreatment on vitrectomy in young patients with proliferative diabetic retinopathy. Ann Thorac Surg. 2020;9 (1):82-89. doi:10.21037/apm.2020.01.10

48. Hainsworth DP, Bebu I, Aiello LP, et al. Risk factors for retinopathy in type 1 diabetes: the DCCT/EDIC Study. Diabetes Care. 2019;42 (5):875-882. doi:10.2337/dc18-2308

49. Wang SY, Andrews CA, Herman WH, Gardner TW, Stein JD. Incidence and risk factors for developing diabetic retinopathy among youths with type 1 or type 2 diabetes throughout the United States. Ophthalmology. 2017;124:424-430. doi:10.1016/j.ophtha.20 16.10.031

50. Safi H, Safi S, Hafezi-Moghadam A, Ahmadieh H. Early detection of diabetic retinopathy. Surv Ophthalmol. 2018;63(5):601-608. doi:10. 1016/j.survophthal.2018.04.003

51. Ellis MP, Lent-Schochet D, Lo T, Yiu G. Emerging concepts in the treatment of diabetic retinopathy. Curr Diab Rep. 2019;19(11). doi:10.1007/s11892-019-1276-5

52. Zheng Y, He M, Congdon N. The worldwide epidemic of diabetic retinopathy. Indian J Ophthalmol. 2012;60(5):428-431. doi:10.4103/ 0301-4738.100542

53. Galiero R, Pafundi PC, Nevola R, et al. The importance of telemedicine during COVID-19 pandemic: a focus on diabetic retinopathy. J Diabetes Res. 2020;2020:1-8. doi:10.1155/2020/9036847

54. Gunasekeran DV, Ting DSW, Tan GSW, Wong TY. Artificial intelligence for diabetic retinopathy screening, prediction and management Curr Opin Ophthalmol. 2020;31(5):357-365. doi:10.1097/ICU.00 00000000000693

55. Bolinger MT, Antonetti DA. Moving past anti-VEGF: novel therapies for treating diabetic retinopathy. Int J Mol Sci. 2016;17(9):1498. doi:10.3390/ijms17091498

56. Zhao Y, Singh RP. The role of anti-vascular endothelial growth factor (anti-VEGF) in the management of proliferative diabetic retinopathy. Drugs Context. 2018;7. doi:10.7573/dic.212532.

57. You Z-P, Zhang Y-L, Shi K, et al. Suppression of diabetic retinopathy with GLUT1 siRNA. Sci Rep. 2017;7(1). doi:10.1038/s41598-01707942-x
58. Mishra M, Duraisamy AJ, Kowluru RA. Sirt1: a guardian of the development of diabetic retinopathy. Diabetes. 2018;67(4):745-754. doi:10.2337/db17-0996

59. Whitehead M, Wickremasinghe S, Osborne A, Van Wijngaarden P, Martin KR. Diabetic retinopathy: a complex pathophysiology requiring novel therapeutic strategies. Expert Opin Biol Ther. 2018;18 (12):1257-1270. doi:10.1080/14712598.2018.1545836

60. Wang JH, Roberts GE, Liu GS. Updates on gene therapy for diabetic retinopathy. Curr Diab Rep. 2020;20(7). doi:10.1007/s11892-02001308-w

61. Nespoux J, Vallon V. SGLT2 inhibition and kidney protection. Clin Sci. 2018;132(12):1329-1339. doi:10.1042/CS20171298

62. Verma S, McMurray JJV. SGLT2 inhibitors and mechanisms of cardiovascular benefit: a state-of-the-art review. Diabetologia. 2018;61(10):2108-2117. doi:10.1007/s00125-018-4670-7

63. Sha W, Wen S, Chen L, Xu B, Lei T, Zhou L. The role of SGLT2 inhibitor on the treatment of diabetic retinopathy. $J$ Diabetes Res. 2020;2020:1-6. doi:10.1155/2020/8867875

64. May M, Framke T, Junker B, Framme C, Pielen A, Schindler C. How and why SGLT2 inhibitors should be explored as potential treatment option in diabetic retinopathy: clinical concept and methodology. Ther Adv Endocrinol Metab. 2019;10. doi:10.1177/2042018819891886.

65. Genentech's faricimab meets primary endpoint and shows strong durability across two global phase III studies for diabetic macular edema, a leading cause of blindness. Genentech; 2020. Available from: https:// www.gene.com/media/press-releases/14891/2020-12-20/genentechsfaricimab-meets-primary-endpo. Accessed May 5, 2021.

66. Mayersohn M. Pharmacokinetics in the elderly. Environ Health Perspect. 1994;102:119-124. doi:10.1289/ehp.94102s11119

67. Turnheim K. Drug therapy in the elderly. Exp Gerontol. 2004;39:1731-1738. doi:10.1016/j.exger.2004.05.011

68. McLean AJ, Le Couteur DG. Aging biology and geriatric clinical pharmacology. Pharmacol Rev. 2004;56(2):163-184. doi:10.1124/ pr.56.2.4

69. Del Amo EM, Rimpelä AK, Heikkinen E, et al. Pharmacokinetic aspects of retinal drug delivery. Prog Retin Eye Res. 2017;57:134-185. doi:10.1016/j.preteyeres.2016.12.001

70. Jackson SHD. Pharmacodynamics in the elderly. J Royal Soc Med. 1994;87:5-7.

71. Andres TM, McGrane T, McEvoy MD, Allen BFS. Geriatric pharmacology: an update. Anesthesiol Clin. 2019;37(3):475-492. doi:10.1016/j.anclin.2019.04.007

72. Mangoni AA, Jackson SHD. Age-related changes in pharmacokinetics and pharmacodynamics: basic principles and practical applications. Br J Clin Pharmacol. 2004;57(1):6-14. doi:10.1046/ j.1365-2125.2003.02007.x

73. Klotz U. Pharmacokinetics and drug metabolism in the elderly. Drug Metab Rev. 2009;41(2):67-76. doi:10.1080/03602530902722679

74. Weinstein JR, Anderson S. The aging kidney: physiological changes. Adv Chronic Kidney Dis. 2010;17(4):302-307. doi:10.1053/j. ackd.2010.05.002

75. Krass I, Schieback P, Dhippayom T. Adherence to diabetes medication: a systematic review. Diabet Med. 2015;32(6):725-737. doi:10.1111/dme.12651

76. Capoccia K, Odegard PS, Letassy N. Medication adherence with diabetes medication: a systematic review of the literature. Diabetes Educ. 2016;42(1):34-71. doi:10.1177/0145721715619038

77. Garoma D, Merga H, Hiko D. Determinants of diabetic retinopathy in Southwest Ethiopia: a Facility-Based Case-Control Study. BMC Public Health. 2020;20(1). doi:10.1186/s12889020-08652-2

78. Rodgers JE, Thudium EM, Beyhaghi H, et al. Predictors of medication adherence in the elderly: the role of mental health. Med Care Res Rev. 2018;75(6):746-761. doi:10.1177/1077558717696992 
79. MacLaughlin EJ, Raehl CL, Treadway AK, Sterling TL, Zoller DP, Bond CA. Assessing medication adherence in the elderly: which tools to use in clinical practice? Drugs Aging. 2005;22(3):231-255. doi:10.2165/00002512-200522030-00005

80. Balkrishnan R. Predictors of medication adherence in the elderly. Clin Ther. 1998;20(4):764-771. doi:10.1016/S0149-2918(98)801 39-2

81. Mohan V, Khunti K, Chan SP, et al. Management of type 2 diabetes in developing countries: balancing optimal glycaemic control and outcomes with affordability and accessibility to treatment. Diabetes Ther. 2020;11(1):15-35. doi:10.1007/s13300-019-00733-9

82. Arlt S, Lindner R, Rösler A, Von Renteln-kruse W. Adherence to medication in patients with dementia: predictors and strategies for improvement. Drugs Aging. 2008;25(12):1033-1047. doi:10.2165/ 0002512-200825120-00005

83. Eppley SE, Mansberger SL, Ramanathan S, Lowry EA. Characteristics associated with adherence to annual dilated eye examinations among us patients with diagnosed diabetes. Ophthalmology. 2019;126(11):1492-1499. doi:10.1016/j.ophtha.2019.05.033

84. Hartnett ME, Key IJ, Loyacano NM, Horswell RL, DeSalvo KB. Perceived barriers to diabetic eye care: Qualitative Study of patients and physicians. Arch Ophthalmol. 2005;123(3):387-391. doi:10.10 01/archopht.123.3.387
85. Angermann R, Rauchegger T, Nowosielski Y, et al. Treatment compliance and adherence among patients with diabetic retinopathy and age-related macular degeneration treated by anti-vascular endothelial growth factor under universal health coverage. Graefes Arch Clin Exp Ophthalmol. 2019;257(10):2119-2125. doi:10.1007/s00417-019-04414-y

86. Obeid A, Su D, Patel SN, et al. Outcomes of eyes lost to follow-up with proliferative diabetic retinopathy that received panretinal photocoagulation versus intravitreal anti-vascular endothelial growth factor. Ophthalmology. 2019;126(3):407-413. doi:10.1016/j.ophtha.20 18.07.027

87. Ciulla TA, Pollack JS, Williams DF. Visual acuity outcomes and anti-VEGF therapy intensity in diabetic macular oedema: a real-world analysis of 28658 patient eyes. $\mathrm{Br}$ $J$ Ophthalmol. 2021;105(2):216-221. doi:10.1136/bjophthalmol2020-315933

88. Ciulla TA, Bracha P, Pollack J, Williams DF. Real-world outcomes of anti-vascular endothelial growth factor therapy in diabetic macular edema in the United States. Ophthalmol Retina. 2018;2 (12):1179-1187. doi:10.1016/j.oret.2018.06.004
Clinical Interventions in Aging

\section{Publish your work in this journal}

Clinical Interventions in Aging is an international, peer-reviewed journal focusing on evidence-based reports on the value or lack thereof of treatments intended to prevent or delay the onset of maladaptive correlates of aging in human beings. This journal is indexed on PubMed Central, MedLine, CAS, Scopus and the Elsevier

\section{Dovepress}

Bibliographic databases. The manuscript management system is completely online and includes a very quick and fair peer-review system, which is all easy to use. Visit http://www.dovepress.com/ testimonials.php to read real quotes from published authors. 